\title{
Klein-Beltrami model. Part IV
}

\author{
Roland Coghetto \\ Rue de la Brasserie 5 \\ 7100 La Louvière, Belgium
}

\begin{abstract}
Summary. Timothy Makarios (with Isabelle/HOL ${ }^{1}$ ) and John Harrison (with HOL-Light ${ }^{2}$ ) shown that "the Klein-Beltrami model of the hyperbolic plane satisfy all of Tarski's axioms except his Euclidean axiom" [2, [3, ,4, 5].

With the Mizar system [1] we use some ideas taken from Tim Makarios's MSc thesis [10 to formalize some definitions and lemmas necessary for the verification of the independence of the parallel postulate. In this article, which is the continuation of [8, we prove that our constructed model satisfies the axioms of segment construction, the axiom of betweenness identity, and the axiom of Pasch due to Tarski, as formalized in [11] and related Mizar articles.
\end{abstract}

MSC: 51A05 51M10 68V20

Keywords: Tarski's geometry axioms; foundations of geometry; Klein-Beltrami model

MML identifier: BKMODEL4, version: 8.1.09 5.60.1371

\section{Preliminaries}

Let us consider real numbers $a, b$. Now we state the propositions:

(1) If $a \neq b$, then $1-\frac{a}{a-b}=-\frac{b}{a-b}$.

(2) If $0<a \cdot b$, then $0<\frac{a}{b}$.

Now we state the propositions:

(3) Let us consider real numbers $a, b, c$. Suppose $0 \leqslant a \leqslant 1$ and $0<b \cdot c$. Then $0 \leqslant \frac{a \cdot c}{(1-a) \cdot b+a \cdot c} \leqslant 1$.

\footnotetext{
${ }^{1}$ https://www.isa-afp.org/entries/Tarskis_Geometry.html

2 https://github.com/jrh13/hol-light/blob/master/100/independence.ml 
(4) Let us consider real numbers $a, b, c$. Suppose $(1-a) \cdot b+a \cdot c \neq 0$. Then $1-\frac{a \cdot c}{(1-a) \cdot b+a \cdot c}=\frac{(1-a) \cdot b}{(1-a) \cdot b+a \cdot c}$.

(5) Let us consider real numbers $a, b, c, d$. If $b \neq 0$, then $\frac{\frac{a \cdot b}{c} \cdot d}{b}=\frac{a \cdot d}{c}$.

(6) Let us consider an element $u$ of $\mathcal{E}_{\mathrm{T}}^{3}$. Then $u=[u(1), u(2), u(3)]$.

(7) Let us consider an element $P$ of the BK-model. Then BK-to-REAL2 $(P) \in$ TarskiEuclid2Space.

Let $P$ be a point of BK-model-Plane. The functor BKtoT2 $(P)$ yielding a point of TarskiEuclid2Space is defined by

(Def. 1) there exists an element $p$ of the BK-model such that $P=p$ and $i t=$ BK-to-REAL2 $(p)$.

Let $P$ be a point of TarskiEuclid2Space. Assume $\hat{P} \in$ the inside of circle $(0,0,1)$. The functor T2toBK $(P)$ yielding a point of BK-model-Plane is defined by

(Def. 2) there exists a non zero element $u$ of $\mathcal{E}_{\mathrm{T}}^{3}$ such that $i t=$ the direction of $u$ and $(u)_{\mathbf{3}}=1$ and $\hat{P}=\left[(u)_{\mathbf{1}},(u)_{\mathbf{2}}\right]$.

Let us consider a point $P$ of BK-model-Plane. Now we state the propositions:

(8) $\operatorname{BKtoT} 2(P) \in$ the inside of circle $(0,0,1)$.

(9) $\mathrm{T} 2 \operatorname{toBK}(\operatorname{BKtoT} 2(P))=P$.

(10) Let us consider a point $P$ of TarskiEuclid2Space. Suppose $\hat{P}$ is an element of the inside of circle $(0,0,1)$. Then BKtoT2 $(\mathrm{T} 2 \operatorname{toBK}(P))=P$.

(11) Let us consider a point $P$ of BK-model-Plane, and an element $p$ of the BK-model. Suppose $P=p$. Then

(i) $\operatorname{BKtoT} 2(P)=$ BK-to-REAL2 $(p)$, and

(ii) $\operatorname{BKto\hat {T}2}(P)=\operatorname{BK}$-to-REAL2 $(p)$.

(12) Let us consider points $P, Q, R$ of BK-model-Plane, and points $P_{2}, Q_{2}, R_{2}$ of TarskiEuclid2Space. Suppose $P_{2}=\operatorname{BKtoT} 2(P)$ and $Q_{2}=\operatorname{BKtoT} 2(Q)$ and $R_{2}=\operatorname{BKtoT} 2(R)$. Then $Q$ lies between $P$ and $R$ if and only if $Q_{2}$ lies between $P_{2}$ and $R_{2}$. The theorem is a consequence of (11).

(13) Let us consider elements $P, Q$ of $\mathcal{E}_{\mathrm{T}}^{2}$. If $P \neq Q$, then $P(1) \neq Q(1)$ or $P(2) \neq Q(2)$.

(14) Let us consider real numbers $a, b$, and elements $P, Q$ of $\mathcal{E}_{\mathrm{T}}^{2}$. If $P \neq Q$ and $(1-a) \cdot P+a \cdot Q=(1-b) \cdot P+b \cdot Q$, then $a=b$. The theorem is a consequence of (13).

(15) Let us consider points $P, Q$ of BK-model-Plane. If BKtoT2 $(P)=$ BKtôT2 $(Q)$, then $P=Q$. The theorem is a consequence of (11).

Let $P, Q, R$ be points of BK-model-Plane. Assume $Q$ lies between $P$ and $R$ and $P \neq R$. The functor length $(P, Q, R)$ yielding a real number is defined by 
(Def. 3$) \quad 0 \leqslant i t \leqslant 1$ and BKtôิT $2(Q)=(1-i t) \cdot(\operatorname{BKtoT} 2(P))+i t \cdot(\operatorname{BKtoT} 2(R))$.

Let us consider points $P, Q$ of BK-model-Plane. Now we state the propositions:

(i) $P$ lies between $P$ and $Q$, and

(ii) $Q$ lies between $P$ and $Q$.

The theorem is a consequence of (12).

(17) If $P \neq Q$, then $\operatorname{length}(P, P, Q)=0$ and $\operatorname{length}(P, Q, Q)=1$. The theorem is a consequence of (16).

(18) Let us consider a square matrix $N$ over $\mathbb{R}_{\mathrm{F}}$ of dimension 3. Suppose $N=\langle\langle 2,0,-1\rangle,\langle 0, \sqrt{3}, 0\rangle,\langle 1,0,-2\rangle\rangle$. Then

(i) $\operatorname{Det} N=(-3) \cdot \sqrt{3}$, and

(ii) $N$ is invertible.

(19) Let us consider elements $a_{11}, a_{12}, a_{13}, a_{21}, a_{22}, a_{23}, a_{31}, a_{32}, a_{33}, b_{11}$, $b_{12}, b_{13}, b_{21}, b_{22}, b_{23}, b_{31}, b_{32}, b_{33}, a_{1}, a_{2}, a_{3}, a_{4}, a_{5}, a_{6}, a_{7}, a_{8}, a_{9}$ of $\mathbb{R}_{\mathrm{F}}$, and square matrices $A, B$ over $\mathbb{R}_{\mathrm{F}}$ of dimension 3 .

Suppose $A=\left\langle\left\langle a_{11}, a_{12}, a_{13}\right\rangle,\left\langle a_{21}, a_{22}, a_{23}\right\rangle,\left\langle a_{31}, a_{32}, a_{33}\right\rangle\right\rangle$ and $B=$ $\left\langle\left\langle b_{11}, b_{12}, b_{13}\right\rangle,\left\langle b_{21}, b_{22}, b_{23}\right\rangle,\left\langle b_{31}, b_{32}, b_{33}\right\rangle\right\rangle$ and $a_{1}=a_{11} \cdot b_{11}+a_{12} \cdot b_{21}+$ $a_{13} \cdot b_{31}$ and $a_{2}=a_{11} \cdot b_{12}+a_{12} \cdot b_{22}+a_{13} \cdot b_{32}$ and $a_{3}=a_{11} \cdot b_{13}+a_{12} \cdot b_{23}+a_{13} \cdot b_{33}$ and $a_{4}=a_{21} \cdot b_{11}+a_{22} \cdot b_{21}+a_{23} \cdot b_{31}$.

Suppose $a_{5}=a_{21} \cdot b_{12}+a_{22} \cdot b_{22}+a_{23} \cdot b_{32}$ and $a_{6}=a_{21} \cdot b_{13}+a_{22} \cdot b_{23}+a_{23}$. $b_{33}$ and $a_{7}=a_{31} \cdot b_{11}+a_{32} \cdot b_{21}+a_{33} \cdot b_{31}$ and $a_{8}=a_{31} \cdot b_{12}+a_{32} \cdot b_{22}+a_{33} \cdot b_{32}$ and $a_{9}=a_{31} \cdot b_{13}+a_{32} \cdot b_{23}+a_{33} \cdot b_{33}$.

Then $A \cdot B=\left\langle\left\langle a_{1}, a_{2}, a_{3}\right\rangle,\left\langle a_{4}, a_{5}, a_{6}\right\rangle,\left\langle a_{7}, a_{8}, a_{9}\right\rangle\right\rangle$.

Let us consider square matrices $N_{1}, N_{2}$ over $\mathbb{R}_{\mathrm{F}}$ of dimension 3 . Now we state the propositions:

(20) Suppose $N_{1}=\langle\langle 2,0,-1\rangle,\langle 0, \sqrt{3}, 0\rangle,\langle 1,0,-2\rangle\rangle$ and $N_{2}=\left\langle\left\langle\frac{2}{3}, 0,-\frac{1}{3}\right\rangle,\langle 0\right.$, $\left.\left.\frac{1}{\sqrt{3}}, 0\right\rangle,\left\langle\frac{1}{3}, 0,-\frac{2}{3}\right\rangle\right\rangle$. Then $N_{1} \cdot N_{2}=\langle\langle 1,0,0\rangle,\langle 0,1,0\rangle,\langle 0,0,1\rangle\rangle$. The theorem is a consequence of (19).

(21) Suppose $N_{2}=\langle\langle 2,0,-1\rangle,\langle 0, \sqrt{3}, 0\rangle,\langle 1,0,-2\rangle\rangle$ and $N_{1}=\left\langle\left\langle\frac{2}{3}, 0,-\frac{1}{3}\right\rangle,\langle 0\right.$, $\left.\left.\frac{1}{\sqrt{3}}, 0\right\rangle,\left\langle\frac{1}{3}, 0,-\frac{2}{3}\right\rangle\right\rangle$. Then $N_{1} \cdot N_{2}=\langle\langle 1,0,0\rangle,\langle 0,1,0\rangle,\langle 0,0,1\rangle\rangle$. The theorem is a consequence of (19).

(22) Suppose $N_{1}=\langle\langle 2,0,-1\rangle,\langle 0, \sqrt{3}, 0\rangle,\langle 1,0,-2\rangle\rangle$ and $N_{2}=\left\langle\left\langle\frac{2}{3}, 0,-\frac{1}{3}\right\rangle,\langle 0\right.$, $\left.\left.\frac{1}{\sqrt{3}}, 0\right\rangle,\left\langle\frac{1}{3}, 0,-\frac{2}{3}\right\rangle\right\rangle$. Then $N_{1}$ is inverse of $N_{2}$. The theorem is a consequence of $(20)$ and (21).

Let us consider an invertible square matrix $N$ over $\mathbb{R}_{\mathrm{F}}$ of dimension 3 . Now we state the propositions: 
(23) Suppose $N=\left\langle\left\langle\frac{2}{3}, 0,-\frac{1}{3}\right\rangle,\left\langle 0, \frac{1}{\sqrt{3}}, 0\right\rangle,\left\langle\frac{1}{3}, 0,-\frac{2}{3}\right\rangle\right\rangle$. Then (the homography of $N)^{\circ}$ (the absolute) $\subseteq$ the absolute.

Proof: (The homography of $N)^{\circ}$ (the absolute) $\subseteq$ the absolute by 7 , (89)], [9, (7)].

(24) Suppose $N=\langle\langle 2,0,-1\rangle,\langle 0, \sqrt{3}, 0\rangle,\langle 1,0,-2\rangle\rangle$. Then (the homography of $N)^{\circ}$ (the absolute) $=$ the absolute.

Proof: (The homography of $N)^{\circ}$ (the absolute) $\subseteq$ the absolute.

The absolute $\subseteq$ (the homography of $N)^{\circ}$ (the absolute) by [6, (19)], (22), (23).

(25) Let us consider real numbers $a, b, r$, and elements $P, Q, R$ of $\mathcal{E}_{T}^{2}$. Suppose $Q \in \mathcal{L}(P, R)$ and $P, R \in$ the inside of $\operatorname{circle}(a, b, r)$. Then $Q \in$ the inside of $\operatorname{circle}(a, b, r)$.

(26) Let us consider non zero elements $u, v$ of $\mathcal{E}_{\mathrm{T}}^{3}$. Suppose the direction of $u=$ the direction of $v$ and $u(3) \neq 0$ and $u(3)=v(3)$. Then $u=v$.

(27) Let us consider an element $R$ of the projective space over $\mathcal{E}_{\mathrm{T}}^{3}$, elements $P, Q$ of the BK-model, non zero elements $u, v, w$ of $\mathcal{E}_{\mathrm{T}}^{3}$, and a real number $r$. Suppose $0 \leqslant r \leqslant 1$ and $P=$ the direction of $u$ and $Q=$ the direction of $v$ and $R=$ the direction of $w$ and $u(3)=1$ and $v(3)=1$ and $w=$ $r \cdot u+(1-r) \cdot v$. Then $R$ is an element of the BK-model.

Proof: Consider $u_{2}$ being a non zero element of $\mathcal{E}_{\mathrm{T}}^{3}$ such that the direction of $u_{2}=P$ and $u_{2}(3)=1$ and BK-to-REAL2 $(P)=\left[u_{2}(1), u_{2}(2)\right] . u=u_{2}$. Reconsider $r_{4}=\left[u_{2}(1), u_{2}(2)\right]$ as an element of $\mathcal{E}_{\mathrm{T}}^{2}$. Consider $v_{2}$ being a non zero element of $\mathcal{E}_{\mathrm{T}}^{3}$ such that the direction of $v_{2}=Q$ and $v_{2}(3)=1$ and BK-to-REAL2 $(Q)=\left[v_{2}(1), v_{2}(2)\right] . v=v_{2}$. Reconsider $r_{6}=\left[v_{2}(1), v_{2}(2)\right]$ as an element of $\mathcal{E}_{\mathrm{T}}^{2}$. Reconsider $r_{8}=[w(1), w(2)]$ as an element of $\mathcal{E}_{\mathrm{T}}^{2}$. $r_{8}=r \cdot r_{4}+(1-r) \cdot r_{6}$. Consider $R_{3}$ being an element of $\mathcal{E}_{\mathrm{T}}^{2}$ such that $R_{3}=r_{8}$ and REAL2-to-BK $\left(r_{8}\right)=$ the direction of $\left[\left(R_{3}\right)_{1},\left(R_{3}\right)_{2}, 1\right]$.

(28) Let us consider an invertible square matrix $N$ over $\mathbb{R}_{\mathrm{F}}$ of dimension 3 , elements $n_{11}, n_{12}, n_{13}, n_{21}, n_{22}, n_{23}, n_{31}, n_{32}, n_{33}$ of $\mathbb{R}_{F}$, points $P, Q$ of the projective space over $\mathcal{E}_{\mathrm{T}}^{3}$, and non zero elements $u, v$ of $\mathcal{E}_{\mathrm{T}}^{3}$. Suppose $N=\left\langle\left\langle n_{11}, n_{12}, n_{13}\right\rangle,\left\langle n_{21}, n_{22}, n_{23}\right\rangle,\left\langle n_{31}, n_{32}, n_{33}\right\rangle\right\rangle$ and $P=$ the direction of $u$ and $Q=$ the direction of $v$ and $Q=($ the homography of $N)(P)$ and $u(3)=1$. Then there exists a non zero real number $a$ such that

(i) $v(1)=a \cdot\left(n_{11} \cdot u(1)+n_{12} \cdot u(2)+n_{13}\right)$, and

(ii) $v(2)=a \cdot\left(n_{21} \cdot u(1)+n_{22} \cdot u(2)+n_{23}\right)$, and

(iii) $v(3)=a \cdot\left(n_{31} \cdot u(1)+n_{32} \cdot u(2)+n_{33}\right)$.

(29) Let us consider an invertible square matrix $N$ over $\mathbb{R}_{\mathrm{F}}$ of dimension 3 , elements $n_{11}, n_{12}, n_{13}, n_{21}, n_{22}, n_{23}, n_{31}, n_{32}, n_{33}$ of $\mathbb{R}_{F}$, an element 
$P$ of the BK-model, a point $Q$ of the projective space over $\mathcal{E}_{\mathrm{T}}^{3}$, and non zero elements $u, v$ of $\mathcal{E}_{\mathrm{T}}^{3}$. Suppose $N=\left\langle\left\langle n_{11}, n_{12}, n_{13}\right\rangle,\left\langle n_{21}, n_{22}, n_{23}\right\rangle,\left\langle n_{31}\right.\right.$, $\left.\left.n_{32}, n_{33}\right\rangle\right\rangle$ and $P=$ the direction of $u$ and $Q=$ the direction of $v$ and $Q=($ the homography of $N)(P)$ and $u(3)=1$ and $v(3)=1$. Then

(i) $n_{31} \cdot u(1)+n_{32} \cdot u(2)+n_{33} \neq 0$, and

(ii) $v(1)=\frac{n_{11} \cdot u(1)+n_{12} \cdot u(2)+n_{13}}{n_{31} \cdot u(1)+n_{32} \cdot u(2)+n_{33}}$, and

(iii) $v(2)=\frac{n_{21} \cdot u(1)+n_{22} \cdot u(2)+n_{23}}{n_{31} \cdot u(1)+n_{32} \cdot u(2)+n_{33}}$.

The theorem is a consequence of (28).

(30) Let us consider an invertible square matrix $N$ over $\mathbb{R}_{\mathrm{F}}$ of dimension 3 , an element $h$ of the subgroup of $K$-isometries, elements $n_{11}, n_{12}, n_{13}$, $n_{21}, n_{22}, n_{23}, n_{31}, n_{32}, n_{33}$ of $\mathbb{R}_{\mathrm{F}}$, an element $P$ of the BK-model, and a non zero element $u$ of $\mathcal{E}_{\mathrm{T}}^{3}$. Suppose $h=$ the homography of $N$ and $N=\left\langle\left\langle n_{11}, n_{12}, n_{13}\right\rangle,\left\langle n_{21}, n_{22}, n_{23}\right\rangle,\left\langle n_{31}, n_{32}, n_{33}\right\rangle\right\rangle$ and $P=$ the direction of $u$ and $u(3)=1$. Then $n_{31} \cdot u(1)+n_{32} \cdot u(2)+n_{33} \neq 0$. The theorem is a consequence of (29).

(31) Let us consider an invertible square matrix $N$ over $\mathbb{R}_{\mathrm{F}}$ of dimension 3 , elements $n_{11}, n_{12}, n_{13}, n_{21}, n_{22}, n_{23}, n_{31}, n_{32}, n_{33}$ of $\mathbb{R}_{F}$, an element $P$ of the absolute, a point $Q$ of the projective space over $\mathcal{E}_{\mathrm{T}}^{3}$, and non zero elements $u, v$ of $\mathcal{E}_{\mathrm{T}}^{3}$. Suppose $N=\left\langle\left\langle n_{11}, n_{12}, n_{13}\right\rangle,\left\langle n_{21}, n_{22}, n_{23}\right\rangle,\left\langle n_{31}\right.\right.$, $\left.\left.n_{32}, n_{33}\right\rangle\right\rangle$ and $P=$ the direction of $u$ and $Q=$ the direction of $v$ and $Q=($ the homography of $N)(P)$ and $u(3)=1$ and $v(3)=1$. Then

(i) $n_{31} \cdot u(1)+n_{32} \cdot u(2)+n_{33} \neq 0$, and

(ii) $v(1)=\frac{n_{11} \cdot u(1)+n_{12} \cdot u(2)+n_{13}}{n_{31} \cdot u(1)+n_{32} \cdot u(2)+n_{33}}$, and

(iii) $v(2)=\frac{n_{21} \cdot u(1)+n_{22} \cdot u(2)+n_{23}}{n_{31} \cdot u(1)+n_{32} \cdot u(2)+n_{33}}$.

The theorem is a consequence of (28).

(32) Let us consider an invertible square matrix $N$ over $\mathbb{R}_{\mathrm{F}}$ of dimension 3 , an element $h$ of the subgroup of $K$-isometries, elements $n_{11}, n_{12}, n_{13}, n_{21}$, $n_{22}, n_{23}, n_{31}, n_{32}, n_{33}$ of $\mathbb{R}_{\mathrm{F}}$, an element $P$ of the absolute, and a non zero element $u$ of $\mathcal{E}_{\mathrm{T}}^{3}$. Suppose $h=$ the homography of $N$ and $N=\left\langle\left\langle n_{11}\right.\right.$, $\left.\left.n_{12}, n_{13}\right\rangle,\left\langle n_{21}, n_{22}, n_{23}\right\rangle,\left\langle n_{31}, n_{32}, n_{33}\right\rangle\right\rangle$ and $P=$ the direction of $u$ and $u(3)=1$. Then $n_{31} \cdot u(1)+n_{32} \cdot u(2)+n_{33} \neq 0$. The theorem is a consequence of $(31)$.

(33) Let us consider an invertible square matrix $N$ over $\mathbb{R}_{\mathrm{F}}$ of dimension 3 , an element $h$ of the subgroup of $K$-isometries, elements $n_{11}, n_{12}, n_{13}$, $n_{21}, n_{22}, n_{23}, n_{31}, n_{32}, n_{33}$ of $\mathbb{R}_{\mathrm{F}}$, an element $P$ of the BK-model, and a non zero element $u$ of $\mathcal{E}_{\mathrm{T}}^{3}$. Suppose $h=$ the homography of $N$ and $N=\left\langle\left\langle n_{11}, n_{12}, n_{13}\right\rangle,\left\langle n_{21}, n_{22}, n_{23}\right\rangle,\left\langle n_{31}, n_{32}, n_{33}\right\rangle\right\rangle$ and $P=$ the direction 
of $u$ and $u(3)=1$. Then (the homography of $N)(P)=$ the direction of $\left[\frac{n_{11} \cdot u(1)+n_{12} \cdot u(2)+n_{13}}{n_{31} \cdot u(1)+n_{32} \cdot u(2)+n_{33}}, \frac{n_{21} \cdot u(1)+n_{22} \cdot u(2)+n_{23}}{n_{31} \cdot u(1)+n_{32} \cdot u(2)+n_{33}}, 1\right]$. The theorem is a consequence of $(29)$.

(34) Let us consider an invertible square matrix $N$ over $\mathbb{R}_{\mathrm{F}}$ of dimension 3 , an element $h$ of the subgroup of $K$-isometries, elements $n_{11}, n_{12}, n_{13}$, $n_{21}, n_{22}, n_{23}, n_{31}, n_{32}, n_{33}$ of $\mathbb{R}_{\mathrm{F}}$, an element $P$ of the absolute, and a non zero element $u$ of $\mathcal{E}_{\mathrm{T}}^{3}$. Suppose $h=$ the homography of $N$ and $N=\left\langle\left\langle n_{11}, n_{12}, n_{13}\right\rangle,\left\langle n_{21}, n_{22}, n_{23}\right\rangle,\left\langle n_{31}, n_{32}, n_{33}\right\rangle\right\rangle$ and $P=$ the direction of $u$ and $u(3)=1$. Then (the homography of $N)(P)=$ the direction of $\left[\frac{n_{11} \cdot u(1)+n_{12} \cdot u(2)+n_{13}}{n_{31} \cdot u(1)+n_{32} \cdot u(2)+n_{33}}, \frac{n_{21} \cdot u(1)+n_{22} \cdot u(2)+n_{23}}{n_{31} \cdot u(1)+n_{32} \cdot u(2)+n_{33}}, 1\right]$. The theorem is a consequence of (31).

(35) Let us consider a subset $A$ of $\mathcal{E}_{\mathrm{T}}^{3}$, a convex, non empty subset $B$ of $\mathcal{E}_{\mathrm{T}}^{2}$, a real number $r$, and an element $x$ of $\mathcal{E}_{\mathrm{T}}^{3}$. Suppose $A=\{x$, where $x$ is an element of $\mathcal{E}_{\mathrm{T}}^{3}:\left[(x)_{\mathbf{1}},(x)_{\mathbf{2}}\right] \in B$ and $\left.(x)_{\mathbf{3}}=r\right\}$. Then $A$ is non empty and convex.

(36) Let us consider elements $n_{1}, n_{2}, n_{3}$ of $\mathbb{R}_{\mathrm{F}}$, and elements $n, u$ of $\mathcal{E}_{\mathrm{T}}^{3}$. Suppose $n=\left\langle n_{1}, n_{2}, n_{3}\right\rangle$ and $u(3)=1$. Then $|(n, u)|=n_{1} \cdot u(1)+n_{2}$. $u(2)+n_{3}$.

(37) Let us consider a convex, non empty subset $A$ of $\mathcal{E}_{\mathrm{T}}^{3}$, and elements $n$, $u, v$ of $\mathcal{E}_{\mathrm{T}}^{3}$. Suppose for every element $w$ of $\mathcal{E}_{\mathrm{T}}^{3}$ such that $w \in A$ holds $|(n, w)| \neq 0$ and $u, v \in A$. Then $0<|(n, u)| \cdot|(n, v)|$.

Proof: Set $x=|(n, u)|$. Set $y=|(n, v)|$. Reconsider $l=\frac{x}{x-y}$ as a non zero real number. Reconsider $w=l \cdot v+(1-l) \cdot u$ as an element of $\mathcal{E}_{\mathrm{T}}^{3} \cdot x \neq y$. $1-l=-\frac{y}{x-y} \cdot|(n, w)|=0$.

Let us consider elements $n_{31}, n_{32}, n_{33}$ of $\mathbb{R}_{\mathrm{F}}$ and elements $u, v$ of $\mathcal{E}_{\mathrm{T}}^{2}$. Now we state the propositions:

(38) Suppose $u, v \in$ the inside of circle $(0,0,1)$ and for every element $w$ of $\mathcal{E}_{\mathrm{T}}^{2}$ such that $w \in$ the inside of circle $(0,0,1)$ holds $n_{31} \cdot w(1)+n_{32} \cdot w(2)+n_{33} \neq 0$. Then $0<\left(n_{31} \cdot u(1)+n_{32} \cdot u(2)+n_{33}\right) \cdot\left(n_{31} \cdot v(1)+n_{32} \cdot v(2)+n_{33}\right)$. The theorem is a consequence of (35), (36), and (37).

(39) Suppose $u \in$ the inside of circle $(0,0,1)$ and $v \in \operatorname{circle}(0,0,1)$ and for every element $w$ of $\mathcal{E}_{\mathrm{T}}^{2}$ such that $w \in$ the closed inside of circle $(0,0,1)$ holds $n_{31} \cdot w(1)+n_{32} \cdot w(2)+n_{33} \neq 0$. Then $0<\left(n_{31} \cdot u(1)+n_{32} \cdot u(2)+\right.$ $\left.n_{33}\right) \cdot\left(n_{31} \cdot v(1)+n_{32} \cdot v(2)+n_{33}\right)$. The theorem is a consequence of $(35)$, (36), and (37).

(40) Let us consider real numbers $l, r$, elements $u, v, w$ of $\mathcal{E}_{\mathrm{T}}^{3}$, and real numbers $n_{11}, n_{12}, n_{13}, n_{21}, n_{22}, n_{23}, n_{31}, n_{32}, n_{33}, m_{1}, m_{2}, m_{3}, m_{4}, m_{5}, m_{6}, m_{7}$, $m_{8}, m_{9}$. 
Suppose $m_{3} \neq 0$ and $m_{6} \neq 0$ and $m_{9} \neq 0$ and $r=\frac{l \cdot m_{6}}{(1-l) \cdot m_{3}+l \cdot m_{6}}$ and $(1-l) \cdot m_{3}+l \cdot m_{6} \neq 0$ and $w=(1-l) \cdot u+l \cdot v$ and $m_{1}=$ $n_{11} \cdot u(1)+n_{12} \cdot u(2)+n_{13}$ and $m_{2}=n_{21} \cdot u(1)+n_{22} \cdot u(2)+n_{23}$ and $m_{3}=n_{31} \cdot u(1)+n_{32} \cdot u(2)+n_{33}$ and $m_{4}=n_{11} \cdot v(1)+n_{12} \cdot v(2)+n_{13}$.

Suppose $m_{5}=n_{21} \cdot v(1)+n_{22} \cdot v(2)+n_{23}$ and $m_{6}=n_{31} \cdot v(1)+n_{32} \cdot v(2)+$ $n_{33}$ and $m_{7}=n_{11} \cdot w(1)+n_{12} \cdot w(2)+n_{13}$ and $m_{8}=n_{21} \cdot w(1)+n_{22} \cdot w(2)+n_{23}$ and $m_{9}=n_{31} \cdot w(1)+n_{32} \cdot w(2)+n_{33}$.

Then $(1-r) \cdot\left[\frac{m_{1}}{m_{3}}, \frac{m_{2}}{m_{3}}, 1\right]+r \cdot\left[\frac{m_{4}}{m_{6}}, \frac{m_{5}}{m_{6}}, 1\right]=\left[\frac{m_{7}}{m_{9}}, \frac{m_{8}}{m_{9}}, 1\right]$. The theorem is a consequence of (4) and (5).

(41) Let us consider an invertible square matrix $N$ over $\mathbb{R}_{F}$ of dimension 3 , an element $h$ of the subgroup of $K$-isometries, elements $n_{11}, n_{12}, n_{13}$, $n_{21}, n_{22}, n_{23}, n_{31}, n_{32}, n_{33}$ of $\mathbb{R}_{\mathrm{F}}$, and an element $P$ of the BK-model. Suppose $h=$ the homography of $N$ and $N=\left\langle\left\langle n_{11}, n_{12}, n_{13}\right\rangle,\left\langle n_{21}, n_{22}\right.\right.$, $\left.\left.n_{23}\right\rangle,\left\langle n_{31}, n_{32}, n_{33}\right\rangle\right\rangle$. Then (the homography of $\left.N\right)(P)=$ the direction of $\left[\frac{n_{11} \cdot(\text { BK-to-REAL2 }(P))_{\mathbf{1}}+n_{12} \cdot(\text { BK-to-REAL2 }(P))_{\mathbf{2}}+n_{13}}{n_{31} \cdot(\text { BK-to-REAL2 }(P))_{1}+n_{32} \cdot(\text { BK-to-REAL2 }(P))_{\mathbf{2}}+n_{33}}\right.$, $\left.\frac{n_{21} \cdot(\text { BK-to-REAL2 }(P))_{\mathbf{1}}+n_{22} \cdot(\text { BK-to-REAL2 }(P))_{\mathbf{2}}+n_{23}}{n_{31} \cdot(\text { BK-to-REAL2 }(P))_{\mathbf{1}}+n_{32} \cdot(\text { BK-to-REAL2 }(P))_{\mathbf{2}}+n_{33}}, 1\right]$.

The theorem is a consequence of (33).

(42) Let us consider an element $h$ of the subgroup of $K$-isometries, an invertible square matrix $N$ over $\mathbb{R}_{\mathrm{F}}$ of dimension 3 , elements $n_{11}, n_{12}, n_{13}$, $n_{21}, n_{22}, n_{23}, n_{31}, n_{32}, n_{33}$ of $\mathbb{R}_{\mathrm{F}}$, and an element $u_{2}$ of $\mathcal{E}_{\mathrm{T}}^{2}$. Suppose $h=$ the homography of $N$ and $N=\left\langle\left\langle n_{11}, n_{12}, n_{13}\right\rangle,\left\langle n_{21}, n_{22}, n_{23}\right\rangle,\left\langle n_{31}\right.\right.$, $\left.\left.n_{32}, n_{33}\right\rangle\right\rangle$ and $u_{2} \in$ the inside of circle $(0,0,1)$. Then $n_{31} \cdot u_{2}(1)+n_{32} \cdot u_{2}(2)+$ $n_{33} \neq 0$. The theorem is a consequence of $(30)$.

(43) Let us consider a positive real number $r$, and an element $u$ of $\mathcal{E}_{\mathrm{T}}^{2}$. If $u \in \operatorname{circle}(0,0, r)$, then $u$ is not zero.

(44) Let us consider an element $h$ of the subgroup of $K$-isometries, an invertible square matrix $N$ over $\mathbb{R}_{\mathrm{F}}$ of dimension 3 , elements $n_{11}, n_{12}, n_{13}$, $n_{21}, n_{22}, n_{23}, n_{31}, n_{32}, n_{33}$ of $\mathbb{R}_{\mathrm{F}}$, and an element $u_{2}$ of $\mathcal{E}_{\mathrm{T}}^{2}$. Suppose $h=$ the homography of $N$ and $N=\left\langle\left\langle n_{11}, n_{12}, n_{13}\right\rangle,\left\langle n_{21}, n_{22}, n_{23}\right\rangle,\left\langle n_{31}\right.\right.$, $\left.\left.n_{32}, n_{33}\right\rangle\right\rangle$ and $u_{2} \in$ the closed inside of circle $(0,0,1)$. Then $n_{31} \cdot u_{2}(1)+$ $n_{32} \cdot u_{2}(2)+n_{33} \neq 0$. The theorem is a consequence of (30), (43), and (32).

(45) Let us consider real numbers $a, b, c, d, e, f, r$. Suppose $(1-r) \cdot[a, b$, $1]+r \cdot[c, d, 1]=[e, f, 1]$. Then $(1-r) \cdot[a, b]+r \cdot[c, d]=[e, f]$.

(46) Let us consider points $P, Q, R, P^{\prime}, Q^{\prime}, R^{\prime}$ of BK-model-Plane, elements $p, q, r, p^{\prime}, q^{\prime}, r^{\prime}$ of the BK-model, an element $h$ of the subgroup of $K$ isometries, and an invertible square matrix $N$ over $\mathbb{R}_{\mathrm{F}}$ of dimension 3 . Suppose $h=$ the homography of $N$ and $Q$ lies between $P$ and $R$ and $P=p$ and $Q=q$ and $R=r$ and $p^{\prime}=$ (the homography of $\left.N\right)(p)$ and $q^{\prime}=($ the homography of $N)(q)$ and $r^{\prime}=($ the homography of $N)(r)$ and 
$P^{\prime}=p^{\prime}$ and $Q^{\prime}=q^{\prime}$ and $R^{\prime}=r^{\prime}$. Then $Q^{\prime}$ lies between $P^{\prime}$ and $R^{\prime}$.

Proof: Consider $n_{11}, n_{12}, n_{13}, n_{21}, n_{22}, n_{23}, n_{31}, n_{32}, n_{33}$ being elements of $\mathbb{R}_{\mathrm{F}}$ such that $N=\left\langle\left\langle n_{11}, n_{12}, n_{13}\right\rangle,\left\langle n_{21}, n_{22}, n_{23}\right\rangle,\left\langle n_{31}, n_{32}, n_{33}\right\rangle\right\rangle$. Consider $u$ being a non zero element of $\mathcal{E}_{\mathrm{T}}^{3}$ such that the direction of $u=p$ and $u(3)=1$ and BK-to-REAL2 $(p)=[u(1), u(2)]$. Consider $v$ being a non zero element of $\mathcal{E}_{\mathrm{T}}^{3}$ such that the direction of $v=r$ and $v(3)=1$ and BK-to-REAL2 $(r)=[v(1), v(2)]$. Consider $w$ being a non zero element of $\mathcal{E}_{\mathrm{T}}^{3}$ such that the direction of $w=q$ and $w(3)=1$ and BK-to-REAL2 $(q)=[w(1), w(2)]$.

Reconsider $m_{1}=n_{11} \cdot u(1)+n_{12} \cdot u(2)+n_{13}, m_{2}=n_{21} \cdot u(1)+n_{22} \cdot$ $u(2)+n_{23}, m_{3}=n_{31} \cdot u(1)+n_{32} \cdot u(2)+n_{33}, m_{4}=n_{11} \cdot v(1)+n_{12} \cdot v(2)+n_{13}$, $m_{5}=n_{21} \cdot v(1)+n_{22} \cdot v(2)+n_{23}, m_{6}=n_{31} \cdot v(1)+n_{32} \cdot v(2)+n_{33}, m_{7}=$ $n_{11} \cdot w(1)+n_{12} \cdot w(2)+n_{13}, m_{8}=n_{21} \cdot w(1)+n_{22} \cdot w(2)+n_{23}, m_{9}=n_{31} \cdot w(1)+$ $n_{32} \cdot w(2)+n_{33}$ as a real number. BKtoT2 $(P)=$ BK-to-REAL2 $(p)$ and $\operatorname{BKtoT} 2(P)=\operatorname{BK}$-to-REAL2 $(p)$ and $\operatorname{BKtoT} 2(Q)=\operatorname{BK}$-to-REAL2 $(q)$ and BKtoT $2(Q)=$ BK-to-REAL2 $(q)$ and BKtoT2 $(R)=$ BK-to-REAL2 $(r)$ and BKtoT $2(R)=$ BK-to-REAL2 $(r)$. Consider $l$ being a real number such that $0 \leqslant l \leqslant 1$ and BKtôि $2(Q)=(1-l) \cdot(\operatorname{BKtoT} 2(P))+l \cdot(\operatorname{BKto\hat {T}} 2(R))$.

Set $r=\frac{l \cdot m_{6}}{(1-l) \cdot m_{3}+l \cdot m_{6}} \cdot(1-r) \cdot\left[\frac{m_{1}}{m_{3}}, \frac{m_{2}}{m_{3}}, 1\right]+r \cdot\left[\frac{m_{4}}{m_{6}}, \frac{m_{5}}{m_{6}}, 1\right]=\left[\frac{m_{7}}{m_{9}}\right.$, $\left.\frac{m_{8}}{m_{9}}, 1\right] .0 \leqslant r \leqslant 1$. BKtoT $2\left(P^{\prime}\right)=\operatorname{BK}$-to-REAL2 $\left(p^{\prime}\right)$ and BKto $\hat{\mathrm{T}} 2\left(P^{\prime}\right)=$ BK-to-REAL2 $\left(p^{\prime}\right)$ and BKtoT2 $\left(Q^{\prime}\right)=\operatorname{BK}$-to-REAL2 $\left(q^{\prime}\right)$ and BKto $\hat{\mathrm{T}} 2\left(Q^{\prime}\right)=$ BK-to-REAL2 $\left(q^{\prime}\right)$ and BKtoT2 $\left(R^{\prime}\right)=\operatorname{BK}$-to-REAL2 $\left(r^{\prime}\right)$ and BKtoT $2\left(R^{\prime}\right)=$ BK-to-REAL2 $\left(r^{\prime}\right)$.

Let $P$ be a point of the projective space over $\mathcal{E}_{\mathrm{T}}^{3}$. We say that $P$ is point at $\infty$ if and only if

(Def. 4) there exists a non zero element $u$ of $\mathcal{E}_{\mathrm{T}}^{3}$ such that $P=$ the direction of $u$ and $(u)_{\mathbf{3}}=0$.

Now we state the proposition:

(47) Let us consider a point $P$ of the projective space over $\mathcal{E}_{\mathrm{T}}^{3}$. Suppose there exists a non zero element $u$ of $\mathcal{E}_{\mathrm{T}}^{3}$ such that $P=$ the direction of $u$ and $(u)_{\mathbf{3}} \neq 0$. Then $P$ is not point at $\infty$.

Note that there exists a point of the projective space over $\mathcal{E}_{\mathrm{T}}^{3}$ which is point at $\infty$ and there exists a point of the projective space over $\mathcal{E}_{\mathrm{T}}^{3}$ which is non point at $\infty$.

Let $P$ be a non point at $\infty$ point of the projective space over $\mathcal{E}_{\mathrm{T}}^{3}$. The functor RP3toREAL2 $(P)$ yielding an element of $\mathcal{R}^{2}$ is defined by

(Def. 5) there exists a non zero element $u$ of $\mathcal{E}_{\mathrm{T}}^{3}$ such that $P=$ the direction of $u$ and $(u)_{\mathbf{3}}=1$ and $i t=\left[(u)_{\mathbf{1}},(u)_{\mathbf{2}}\right]$. 
The functor RP3toT2 $(P)$ yielding a point of TarskiEuclid2Space is defined by the term

(Def. 6) RP3toREAL2 $(P)$.

Now we state the propositions:

(48) Let us consider non point at $\infty$ elements $P, Q, R, P^{\prime}, Q^{\prime}, R^{\prime}$ of the projective space over $\mathcal{E}_{\mathrm{T}}^{3}$, an element $h$ of the subgroup of $K$-isometries, and an invertible square matrix $N$ over $\mathbb{R}_{\mathrm{F}}$ of dimension 3 .

Suppose $h=$ the homography of $N$ and $P, Q \in$ the BK-model and $R \in$ the absolute and $P^{\prime}=($ the homography of $N)(P)$ and $Q^{\prime}=$ (the homography of $N)(Q)$ and $R^{\prime}=($ the homography of $N)(R)$ and RP3toT2 $(Q)$ lies between RP3toT2 $(P)$ and RP3toT2 $(R)$.

Then RP3toT2 $\left(Q^{\prime}\right)$ lies between RP3toT2 $\left(P^{\prime}\right)$ and RP3toT2 $\left(R^{\prime}\right)$.

PROOF: Consider $n_{11}, n_{12}, n_{13}, n_{21}, n_{22}, n_{23}, n_{31}, n_{32}, n_{33}$ being elements of $\mathbb{R}_{\mathrm{F}}$ such that $N=\left\langle\left\langle n_{11}, n_{12}, n_{13}\right\rangle,\left\langle n_{21}, n_{22}, n_{23}\right\rangle,\left\langle n_{31}, n_{32}, n_{33}\right\rangle\right\rangle$. Consider $u$ being a non zero element of $\mathcal{E}_{\mathrm{T}}^{3}$ such that $P=$ the direction of $u$ and $(u)_{\mathbf{3}}=1$ and RP3toREAL2 $(P)=\left[(u)_{\mathbf{1}},(u)_{\mathbf{2}}\right]$. Consider $v$ being a non zero element of $\mathcal{E}_{\mathrm{T}}^{3}$ such that $R=$ the direction of $v$ and $(v)_{\mathbf{3}}=1$ and RP3toREAL2 $(R)=\left[(v)_{\mathbf{1}},(v)_{\mathbf{2}}\right]$. Consider $w$ being a non zero element of $\mathcal{E}_{\mathrm{T}}^{3}$ such that $Q=$ the direction of $w$ and $(w)_{\mathbf{3}}=1$ and $\operatorname{RP} 3$ toREAL2 $(Q)=\left[(w)_{\mathbf{1}},(w)_{\mathbf{2}}\right]$.

Reconsider $m_{1}=n_{11} \cdot u(1)+n_{12} \cdot u(2)+n_{13}, m_{2}=n_{21} \cdot u(1)+n_{22} \cdot u(2)+$ $n_{23}, m_{3}=n_{31} \cdot u(1)+n_{32} \cdot u(2)+n_{33}, m_{4}=n_{11} \cdot v(1)+n_{12} \cdot v(2)+n_{13}$, $m_{5}=n_{21} \cdot v(1)+n_{22} \cdot v(2)+n_{23}, m_{6}=n_{31} \cdot v(1)+n_{32} \cdot v(2)+n_{33}$, $m_{7}=n_{11} \cdot w(1)+n_{12} \cdot w(2)+n_{13}, m_{8}=n_{21} \cdot w(1)+n_{22} \cdot w(2)+n_{23}$, $m_{9}=n_{31} \cdot w(1)+n_{32} \cdot w(2)+n_{33}$ as a real number.

Consider $l$ being a real number such that $0 \leqslant l \leqslant 1$ and $\operatorname{RP} 3 \operatorname{toT} 2(Q)=$ $(1-l) \cdot(\mathrm{RP} 3 \mathrm{toT} 2(P))+l \cdot(\mathrm{RP} 3 \mathrm{toT} 2(R))$. Set $r=\frac{l \cdot m_{6}}{(1-l) \cdot m_{3}+l \cdot m_{6}} \cdot(1-r) \cdot\left[\frac{m_{1}}{m_{3}}\right.$, $\left.\frac{m_{2}}{m_{3}}, 1\right]+r \cdot\left[\frac{m_{4}}{m_{6}}, \frac{m_{5}}{m_{6}}, 1\right]=\left[\frac{m_{7}}{m_{9}}, \frac{m_{8}}{m_{9}}, 1\right] .0 \leqslant r \leqslant 1$.

(49) Let us consider real numbers $a, b, c$, and elements $u, v, w$ of $\mathcal{E}_{\mathrm{T}}^{3}$. Suppose $a \neq 0$ and $a+b+c=0$ and $a \cdot u+b \cdot v+c \cdot w=0_{\mathcal{E}_{\mathrm{T}}^{3}}$. Then $u \in \operatorname{Line}(v, w)$.

(50) Let us consider non point at $\infty$ points $P, Q, R$ of the projective space over $\mathcal{E}_{\mathrm{T}}^{3}$, and non zero elements $u, v, w$ of $\mathcal{E}_{\mathrm{T}}^{3}$. Suppose $P=$ the direction of $u$ and $Q=$ the direction of $v$ and $R=$ the direction of $w$ and $(u)_{\mathbf{3}}=1$ and $(v)_{\mathbf{3}}=1$ and $(w)_{\mathbf{3}}=1$. Then $P, Q$ and $R$ are collinear if and only if $u, v$ and $w$ are collinear. The theorem is a consequence of (49).

(51) Let us consider elements $u, v, w$ of $\mathcal{E}_{\mathrm{T}}^{3}$. Suppose $u \in \mathcal{L}(v, w)$. Then $\left[(u)_{1}\right.$, $\left.(u)_{\mathbf{2}}\right] \in \mathcal{L}\left(\left[(v)_{\mathbf{1}},(v)_{\mathbf{2}}\right],\left[(w)_{\mathbf{1}},(w)_{\mathbf{2}}\right]\right)$.

(52) Let us consider elements $u, v, w$ of $\mathcal{E}_{\mathrm{T}}^{2}$. Suppose $u \in \mathcal{L}(v, w)$. Then $\left[(u)_{\mathbf{1}}\right.$, $\left.(u)_{\mathbf{2}}, 1\right] \in \mathcal{L}\left(\left[(v)_{\mathbf{1}},(v)_{\mathbf{2}}, 1\right],\left[(w)_{\mathbf{1}},(w)_{\mathbf{2}}, 1\right]\right)$. 
Proof: Consider $r$ being a real number such that $0 \leqslant r$ and $r \leqslant 1$ and $u=(1-r) \cdot v+r \cdot w$. Reconsider $u^{\prime}=\left[(u)_{\mathbf{1}},(u)_{\mathbf{2}}, 1\right], v^{\prime}=\left[(v)_{\mathbf{1}},(v)_{\mathbf{2}}, 1\right]$, $w^{\prime}=\left[(w)_{\mathbf{1}},(w)_{\mathbf{2}}, 1\right]$ as an element of $\mathcal{E}_{\mathrm{T}}^{3} \cdot u^{\prime}=(1-r) \cdot v^{\prime}+r \cdot w^{\prime}$.

(53) Let us consider non point at $\infty$ points $P, Q, R$ of the projective space over $\mathcal{E}_{\mathrm{T}}^{3}$. Then $P, Q$ and $R$ are collinear if and only if RP3toT2 $(P)$, RP3toT2 $(Q)$ and RP3toT2 $(R)$ are collinear. The theorem is a consequence of (50), (51), and (52).

(54) Let us consider elements $u, v, w$ of $\mathcal{E}_{\mathrm{T}}^{2}$. Suppose $u, v$ and $w$ are collinear. Then $\left[(u)_{\mathbf{1}},(u)_{\mathbf{2}}, 1\right],\left[(v)_{\mathbf{1}},(v)_{\mathbf{2}}, 1\right]$ and $\left[(w)_{\mathbf{1}},(w)_{\mathbf{2}}, 1\right]$ are collinear. The theorem is a consequence of $(52)$.

(55) Let us consider non point at $\infty$ elements $P, Q, P_{1}$ of the projective space over $\mathcal{E}_{\mathrm{T}}^{3}$. Suppose $P, Q \in$ the BK-model and $P_{1} \in$ the absolute. Then RP3toT2 $\left(P_{1}\right)$ does not lie between RP3toT2 $(Q)$ and RP3toT2 $(P)$. The theorem is a consequence of (52) and (27).

The functor Dir001 yielding a non point at $\infty$ element of the projective space over $\mathcal{E}_{\mathrm{T}}^{3}$ is defined by the term

(Def. 7) the direction of $[0,0,1]$.

The functor Dir101 yielding a non point at $\infty$ element of the projective space over $\mathcal{E}_{\mathrm{T}}^{3}$ is defined by the term

(Def. 8) the direction of $[1,0,1]$.

Now we state the propositions:

(56) Let us consider non point at $\infty$ elements $P, Q$ of the projective space over $\mathcal{E}_{\mathrm{T}}^{3}$. Suppose $P, Q \in$ the absolute. Then $\overline{\mathrm{RP} 3 t o T 2(\operatorname{Dir} 001) \operatorname{RP} 3 \operatorname{toT} 2(P)} \cong$ RP3toT2(Dir001) RP3toT2( $Q)$.

(57) Let us consider non point at $\infty$ elements $P, Q, R$ of the projective space over $\mathcal{E}_{\mathrm{T}}^{3}$, and non zero elements $u, v, w$ of $\mathcal{E}_{\mathrm{T}}^{3}$. Suppose $P, Q \in$ the absolute and $P \neq Q$ and $P=$ the direction of $u$ and $Q=$ the direction of $v$ and $R=$ the direction of $w$ and $(u)_{\mathbf{3}}=1$ and $(v)_{\mathbf{3}}=1$ and $w=\left[\frac{(u)_{\mathbf{1}}+(v)_{\mathbf{1}}}{2}\right.$, $\left.\frac{(u)_{\mathbf{2}}+(v)_{\mathbf{2}}}{2}, 1\right]$. Then $R \in$ the BK-model.

Proof: Reconsider $u^{\prime}=[u(1), u(2)], v^{\prime}=[v(1), v(2)]$ as an element of $\mathcal{E}_{\mathrm{T}}^{2} \cdot u^{\prime} \neq v^{\prime}$. Reconsider $r_{8}=\left[(w)_{1},(w)_{\mathbf{2}}\right]$ as an element of the inside of circle $(0,0,1)$. Consider $R_{3}$ being an element of $\mathcal{E}_{\mathrm{T}}^{2}$ such that $R_{3}=r_{8}$ and REAL2-to-BK $\left(r_{8}\right)=$ the direction of $\left[\left(R_{3}\right)_{\mathbf{1}},\left(R_{3}\right)_{\mathbf{2}}, 1\right]$.

(58) Let us consider points $R_{1}, R_{2}$ of TarskiEuclid2Space. Suppose $\hat{R}_{1}, \hat{R}_{2} \in$ circle $(0,0,1)$ and $R_{1} \neq R_{2}$. Then there exists an element $P$ of BK-modelPlane such that BKtoT2 $(P)$ lies between $R_{1}$ and $R_{2}$. The theorem is a consequence of (47), (57), and (26).

(59) Let us consider non point at $\infty$ elements $P, Q$ of the projective space 
over $\mathcal{E}_{\mathrm{T}}^{3}$. If $\operatorname{RP} 3 \operatorname{toT} 2(P)=\operatorname{RP} 3 \operatorname{toT} 2(Q)$, then $P=Q$.

(60) Let us consider non point at $\infty$ elements $R_{1}, R_{2}$ of the projective space over $\mathcal{E}_{\mathrm{T}}^{3}$. Suppose $R_{1}, R_{2} \in$ the absolute and $R_{1} \neq R_{2}$. Then there exists an element $P$ of BK-model-Plane such that BKtoT2 $(P)$ lies between RP3toT2 $\left(R_{1}\right)$ and RP3toT2 $\left(R_{2}\right)$. The theorem is a consequence of (59) and (58).

(61) Let us consider points $P, Q, R$ of TarskiEuclid2Space. Suppose $Q$ lies between $P$ and $R$ and $\hat{P}, \hat{R} \in$ the inside of circle $(0,0,1)$. Then $\hat{Q} \in$ the inside of circle $(0,0,1)$.

Let us consider a non point at $\infty$ element $P$ of the projective space over $\mathcal{E}_{\mathrm{T}}^{3}$.

(62) If $P \in$ the absolute, then RP3toREAL2 $(P) \in \operatorname{circle}(0,0,1)$.

(63) If $P \in$ the BK-model, then RP3toREAL2 $(P) \in$ the inside of circle $(0,0,1)$. The theorem is a consequence of (26).

(64) Let us consider a non point at $\infty$ point $P$ of the projective space over $\mathcal{E}_{\mathrm{T}}^{3}$, and an element $Q$ of the BK-model. If $P=Q$, then RP3toREAL2 $(P)=$ BK-to-REAL2 $(Q)$. The theorem is a consequence of (26).

(65) Let us consider non point at $\infty$ elements $P, Q, R_{1}, R_{2}$ of the projective space over $\mathcal{E}_{\mathrm{T}}^{3}$. Suppose $P \neq Q$ and $P \in$ the BK-model and $R_{1}, R_{2} \in$ the absolute and RP3toT2 $(Q)$ lies between RP3toT2 $(P)$ and RP3toT2 $\left(R_{1}\right)$ and RP3toT2 $(Q)$ lies between RP3toT2 $(P)$ and RP3toT2 $\left(R_{2}\right)$. Then $R_{1}=$ $R_{2}$. The theorem is a consequence of (60), (59), (62), (64), (8), and (61).

(66) Let us consider non point at $\infty$ elements $P, Q, P_{1}, P_{2}$ of the projective space over $\mathcal{E}_{\mathrm{T}}^{3}$. Suppose $P \neq Q$ and $P, Q \in$ the BK-model and $P_{1}, P_{2} \in$ the absolute and $P_{1} \neq P_{2}$ and $P, Q$ and $P_{1}$ are collinear and $P, Q$ and $P_{2}$ are collinear. Then

(i) RP3toT2 $(P)$ lies between RP3toT2 $(Q)$ and RP3toT2 $\left(P_{1}\right)$, or

(ii) RP3toT2 $(P)$ lies between RP3toT2 $(Q)$ and RP3toT2 $\left(P_{2}\right)$.

The theorem is a consequence of (55), (53), and (65).

Let us consider elements $P, Q$ of the BK-model. Now we state the propositions:

(67) Suppose $P \neq Q$. Then there exists an element $R$ of the absolute such that for every non point at $\infty$ elements $p, q, r$ of the projective space over $\mathcal{E}_{\mathrm{T}}^{3}$ such that $p=P$ and $q=Q$ and $r=R$ holds RP3toT2( $\left.p\right)$ lies between RP3toT2 $(q)$ and RP3toT2 $(r)$. The theorem is a consequence of (47) and (66).

(68) Suppose $P \neq Q$. Then there exists an element $R$ of the absolute such that for every non point at $\infty$ elements $p, q, r$ of the projective space over 
$\mathcal{E}_{\mathrm{T}}^{3}$ such that $p=P$ and $q=Q$ and $r=R$ holds RP3toT2 $(q)$ lies between RP3toT2 $(p)$ and RP3toT2 $(r)$. The theorem is a consequence of (67).

(69) The direction of $[1,0,1]$ is an element of the absolute.

(70) Let us consider points $a, b$ of BK-model-Plane. Then $\overline{a a} \cong \overline{b b}$. The theorem is a consequence of (69).

(71) Every element of the BK-model is a non point at $\infty$ element of the projective space over $\mathcal{E}_{\mathrm{T}}^{3}$. The theorem is a consequence of (47).

(72) Every element of the absolute is a non point at $\infty$ element of the projective space over $\mathcal{E}_{\mathrm{T}}^{3}$. The theorem is a consequence of (47).

(73) Let us consider an element $P$ of the BK-model, and a non point at $\infty$ element $P^{\prime}$ of the projective space over $\mathcal{E}_{\mathrm{T}}^{3}$. If $P=P^{\prime}$, then RP3toREAL2 $\left(P^{\prime}\right)=$ BK-to-REAL2 $(P)$. The theorem is a consequence of $(26)$.

(74) Let us consider points $a, q, b, c$ of BK-model-Plane. Then there exists a point $x$ of BK-model-Plane such that

(i) $a$ lies between $q$ and $x$, and

(ii) $\overline{a x} \cong \overline{b c}$.

The theorem is a consequence of (71), (68), (72), (12), (70), (48), and (73).

(75) Let us consider points $P, Q$ of BK-model-Plane.

If BKtoT2 $(P)=\operatorname{BKtoT2}(Q)$, then $P=Q$.

(76) Let us consider real numbers $a, b, r$, and elements $P, Q, R$ of $\mathcal{E}_{\mathrm{T}}^{2}$. Suppose $P, R \in$ the inside of $\operatorname{circle}(a, b, r)$. Then $\mathcal{L}(P, R) \subseteq$ the inside of $\operatorname{circle}(a, b, r)$.

\section{The Axiom of Segment Construction}

Now we state the proposition:

(77) BK-model-Plane satisfies the axiom of segment construction.

\section{The Axiom of Betweenness Identity}

Now we state the proposition:

(78) BK-model-Plane satisfies the axiom of betweenness identity. The theorem is a consequence of (12) and (75). 


\section{The Axiom of Pasch}

Now we state the proposition:

(79) BK-model-Plane satisfies the axiom of Pasch. The theorem is a consequence of (12), (8), (25), and (10).

\section{REFERENCES}

[1] Grzegorz Bancerek, Czesław Byliński, Adam Grabowski, Artur Korniłowicz, Roman Matuszewski, Adam Naumowicz, Karol Pąk, and Josef Urban. Mizar: State-of-the-art and beyond. In Manfred Kerber, Jacques Carette, Cezary Kaliszyk, Florian Rabe, and Volker Sorge, editors, Intelligent Computer Mathematics, volume 9150 of Lecture Notes in Computer Science, pages 261-279. Springer International Publishing, 2015. ISBN 978-3319-20614-1. doi:10.1007/978-3-319-20615-8_17.

[2] Eugenio Beltrami. Saggio di interpetrazione della geometria non-euclidea. Giornale di Matematiche, 6:284-322, 1868.

[3] Eugenio Beltrami. Essai d'interprétation de la géométrie non-euclidéenne. In Annales scientifiques de l'École Normale Supérieure. Trad. par J. Hoüel, volume 6, pages 251288. Elsevier, 1869.

[4] Karol Borsuk and Wanda Szmielew. Foundations of Geometry. North Holland, 1960.

[5] Karol Borsuk and Wanda Szmielew. Podstawy geometrii. Państwowe Wydawnictwo Naukowe, Warszawa, 1955 (in Polish).

[6] Roland Coghetto. Homography in $\mathbb{R P}^{2}$. Formalized Mathematics, 24(4):239-251, 2016. doi:10.1515/forma-2016-0020.

[7] Roland Coghetto. Klein-Beltrami model. Part I. Formalized Mathematics, 26(1):21-32, 2018. doi $10.2478 /$ forma-2018-0003.

[8] Roland Coghetto. Klein-Beltrami model. Part III. Formalized Mathematics, 28(1):1-7, 2020. doi $10.2478 /$ forma-2020-0001.

[9] Kanchun, Hiroshi Yamazaki, and Yatsuka Nakamura. Cross products and tripple vector products in 3-dimensional Euclidean space. Formalized Mathematıcs, 11(4):381-383, 2003.

[10] Timothy James McKenzie Makarios. A mechanical verification of the independence of Tarski's Euclidean Axiom. Victoria University of Wellington, New Zealand, 2012. Master's thesis.

[11] William Richter, Adam Grabowski, and Jesse Alama. Tarski geometry axioms. Formalized Mathematics, 22(2):167-176, 2014. doi 10.2478/forma-2014-0017.

Accepted December 30, 2019 